\title{
Efeito de uma intervenção cognitivo- motora sobre os sintomas depressivos de pacientes com doença de Parkinson
}

\author{
Effects of a cognitive-motor intervention on depressive \\ symptoms in patients with Parkinson's disease
}

Gustavo Christofoletti ’, Evandro Rocha Cândido, Larissa Olmedo', Suzi Rosa Barbosa Miziara', Fernanda Beinotti ${ }^{4}$

\section{RESUMO}

Objetivo: Verificar a eficácia de uma intervenção cognitivo-motora sobre os sintomas depressivos de pacientes com DP idiopática. Métodos: Foi realizado um estudo longitudinal, composto por 26 pacientes, divididos entre os grupos experimental e controle. Os sujeitos foram avaliados pela Escala de Depressão Geriátrica, sendo o grupo experimental submetido a uma sequência de exercícios fisioterápicos de estimulação cognitivo-motora durante seis meses. Para a análise dos dados, foram aplicados os testes de Análise de Variâncias para medidas repetidas, a estatística por contraste e o teste $t$ de Student para amostras independentes, sob um nível de significância de 5\%. Resultados: Os resultados apontaram homogeneidade dos grupos no momento inicial, e o grupo submetido ao tratamento fisioterápico obteve uma tendência de significância na interação "momento de intervenção" versus "grupo", evidenciado pelo erro alfa de 6\%, com contraste linear de dados. Con-

\section{Palavras-chave}

Doença de Parkinson, fisioterapia, ensaio clínico, saúde do idoso.

clusão: $O$ protocolo proposto apresentou boa aceitação pelos pacientes com DP. Apesar de não termos alcançado um intervalo de confiança de 95\%, a tendência de significância obtida vislumbra um potencial do referido protocolo, mas com a necessidade de maiores estudos comprobatórios.

\section{ABSTRACT}

Objective: To verify the effectiveness of a cognitive-motor intervention on depressive symptoms in idiopathic PD patients. Methods: This longitudinal design study assessed 26 patients, divided into experimental and control groups. The subjects were evaluated by means of the Geriatric Depression Scale, being, the experimental group, submitted to a six-month protocol of physiotherapeutic exercises on motor and cognitive functions. With respect to the data analysis, were applied the Repeated Measure Analysis of Variance, the contrast statistic and the Student-t test for independent samples, with a significant level of 5\%. Results: The results showed homogeneity of the groups at baseline and the experimental group achieved a trend of significance in the interaction "moment of inter-

1 Universidade Federal de Mato Grosso do Sul (UFMS), Curso de Fisioterapia.

2 Programa de Pós-graduação Stricto-Sensu em Saúde e Desenvolvimento do Centro-Oeste.

3 Universidade Estadual de Goiás (UEG).

4 Universidade Estadual de Campinas (Unicamp), Faculdade de Ciências Médicas.

Endereço para correspondência: Gustavo Christofoletti 


\section{Keywords}

Parkinson's disease, physical therapy, clinical trial, health of the elderly. vention versus group", as evidenced by the alpha error of $6 \%$ with a linear contrast data curve. Conclusion: The proposed protocol has been well accepted by patients diagnosed with idiopathic PD. Although we couldn't contemplate an interval of confidence of 95\%, the significant trend obtained represents a potential of this protocol, but with a need of further corroborative studies.

\section{INTRODUÇÃO}

A doença de Parkinson (DP) é uma doença neurodegenerativa progressiva que afeta $0,3 \%$ da população e apresenta maior incidência entre os homens. É uma afecção comum em idosos e representa um grave problema de saúde pública, por apresentar prevalência crescente de $1 \%$ aos 60 anos para 4\% na população acima de 80 anos $^{1}$.

Classicamente, a DP caracteriza-se por tremor em repouso, rigidez, hipertonia plástica, bradicinesia e grave comprometimento do equilíbrio². Contudo, os sintomas motores clássicos coexistem com prejuízo nos domínios cognitivos, variando de déficits sutis de memória e atenção, a evidente quadro demencial ${ }^{3}$. Trata-se de uma doença progressiva, de evolução crônica, de etiologia idiopática e altamente incapacitante.

Em pacientes com DP, as vias dopaminérgicas sofrem degeneração, levando a déficits de neurotransmissores nas sinapses do sistema nervoso central ${ }^{3}$. A queda nas taxas de dopamina se dá por um processo de apoptose de neurônios da pars compacta mesencefálica, associada à formação de agregados fibrilares compostos pela proteína a-sinucleína ${ }^{4,5}$.

Conforme relatam McKinlay et al. ${ }^{6}$, outros sinais que podem ocorrer concomitantemente à evolução da DP envolvem sintomas neuropsiquiátricos. Para esses autores, mais de 77\% de pacientes analisados relataram apresentar algum distúrbio neuropsiquiátrico, sendo os sintomas depressivos os mais característicos ${ }^{7}$. Stella et al. ${ }^{8}$ corroboram o estudo anterior, demonstrando que depressão, disforia, ansiedade e mudanças de apetite são sintomas altamente frequentes na DP.

Particularmente no que se refere à depressão, estudos comprovam uma importante associação dessa variável com declínio motor na DP, gerando piores escores na realização das atividades instrumentais da vida diária9 . Dickson et al. ${ }^{10}$ argumentam que, enquanto o substrato anatômico da depressão na DP ainda não esteja completamente elucidado, há indícios de comprometimento dos neurotransmissores norepinefrina e serotonina, causando os sintomas "não motores" característicos. É por causa do comprometimento precoce no núcleo ceruleus e no núcleo da rafe que ocorre a associação entre os distúrbios psiquiátricos e motores, potencializando a incapacidade física do paciente ${ }^{10}$.

Diante de tal quadro, uma série de abordagens farmacológicas e não farmacológicas vem sendo proposta, visando promover uma melhora na qualidade de vida do paciente. Alterações endógenas, como liberação de neurotransmissores, modificação no fluxo sanguíneo cerebral e potencializa- ção da neurogênese e de fatores neurotróficos, representam alguns dos processos estimulados com a realização de exercício físico ${ }^{11}$.

Conquanto muitos estudos foquem sua atuação sobre os sintomas motores da DP, as variáveis neuropsiquiátricas são de grande relevância clínica e social e não podem ser negligenciadas. Assim sendo, o objetivo deste trabalho foi verificar a eficácia de uma intervenção cognitivo-motora sobre os sintomas depressivos de pacientes com DP.

\section{MÉTODOS}

Para alcançar os objetivos propostos, foi realizado um estudo longitudinal, do tipo ensaio clínico, formado por dois grupos independentes, a constar: grupo experimental (GE, formado por 13 participantes) e grupo controle (GC, composto por 13 sujeitos).

No que se refere à seleção dos indivíduos, foram admitidos sujeitos diagnosticados com DP idiopática12, com idade mínima de 60 anos, de ambos os sexos, diferentes credos e graus de escolaridade, acompanhados no Ambulatório de Neurociências do Hospital das Clínicas da Universidade Federal de Goiás, e que foram classificados pelo corpo clínico como tendo algum sintoma depressivo relacionado. A aleatorização da amostra ocorreu nesse momento, a partir dos sujeitos que tinham interesse e disponibilidade em participar do trabalho. Em ambos os grupos foram incluídos apenas os participantes que se encontravam nos estágios de 2 a 4 na escala de Hoehn e Yarh ${ }^{13}$ e com a classificação moderada no subescore motor da Escala Unificada de Classificação da DP (Unified Parkinson's Disease Rating Scale) ${ }^{14}$. Foram excluídos do estudo os pacientes que já realizavam atividade física regular ou outra terapia motora, aqueles que apresentavam alguma comorbidade associada à DP, os drogaditos, alcoólatras e aqueles que faziam uso de neurolépticos. Nos casos em que foi encontrado algum indicativo de déficit cognitivo (evidenciado pelo Miniexame do Estado Mental ${ }^{15}$, Bateria Breve de Rastreio Cognitivo ${ }^{16}$ e Teste do Desenho do Relógio ${ }^{17}$ ), os pacientes foram novamente encaminhados ao Ambulatório de Neurociências do Hospital das Clínicas da Universidade Federal de Goiás e, em caso positivo de diagnóstico demencial, foram excluídos do trabalho. Por fim, os pacientes previamente diagnosticados com depressão maior segundo os critérios DSM-IV-TR ${ }^{18}$, e os que já faziam uso de medicação antidepressiva também foram excluídos 
da amostra, para que o foco desta pesquisa se concentrasse nos sintomas depressivos, e não na doença em si.

O tratamento proposto aos pacientes do GE consistiu na realização de uma assistência em grupo, promovida por uma equipe de fisioterapeutas (docentes, discentes e colaboradores), durante seis meses. Em sessões de 1 hora com frequência de três dias na semana, os participantes foram submetidos a exercícios que estimulassem as funções cognitivas e motoras. Os materiais utilizados na terapia consistiram de bolas suíças de todos os tamanhos, além de bolas esportivas (cada qual com seu peso específico), tábuas de equilíbrio, bastões, fitas adesivas e colchonetes.

A terapia foi dividida de forma que fossem realizadas tarefas específicas em cada dia da semana. Em todos os dias, a sessão era iniciada com alongamentos de membros superiores, membros inferiores e tronco. Ao final de cada sessão, realizavam-se atividades lúdicas, associadas a exercícios de dissociação de cinturas escapular e pélvica, propriocepção e tarefas que estimulassem as funções cognitivas, como a atenção concentrada e a memória. Como exemplo das atividades cognitivo-motoras desenvolvidas, podemos citar a tarefa de passar uma bola para cada participante do grupo, quando este deveria dizer substantivos iniciados com determinadas letras do alfabeto, realizar contas matemáticas, identificar objetos e cores, além de falar nomes de frutas, alimentos, cidades ou do colega ao lado.

A parte motora de cada sessão era subdividida da seguinte forma: no primeiro dia de tratamento da semana, eram enfatizados exercícios envolvendo a técnica de facilitação neuromuscular proprioceptiva. Os exercícios eram alternados, visando estimular as diversas posturas. No meio da semana, eram trabalhadas atividades "com bola suíça" e rolos, enfatizando a rotação de tronco e transferências. Na última sessão da semana, a meta era estimular o equilíbrio dinâmico dos pacientes; assim sendo, os exercícios consistiam em percorrer circuitos específicos que exigissem a deambulação em linha reta e em curvas, com e sem obstáculos. Apesar de o tratamento fisioterápico ter sido voltado aos pacientes com DP idiopática que apresentavam sintomas depressivos, foram aceitos familiares, amigos e cuidadores que desejassem participar da terapia.

Em relação ao GC, não houve nenhuma alteração nas atividades motoras basais dos sujeitos. A medicação administrada aos participantes foi mantida nos dois grupos, sendo registrada no início e no final do estudo. A mudança em dosagem ou princípio ativo de medicação culminou na exclusão do sujeito. Todos os participantes foram avaliados na fase on da medicação.

No que se refere aos instrumentos de coleta dados, foi aplicado um questionário geral, com objetivo de caracterizar a amostra (nome, idade, gênero, gravidade da doença, nível de escolaridade e aspectos socioeconômicos). Além do questionário, foi aplicada a Escala de Depressão Geriátrica $(E D G)^{19}$, para quantificar os sintomas depressivos dos sujeitos no início e no final do tratamento. A EDG é um dos instrumentos mais frequentemente utilizados para o rastreamento de sintomas depressivos em idosos. A nota de corte da escala varia de 0 a 30 pontos e, segundo Souza et al.20, tal instrumento apresenta melhor sensibilidade e fidedignidade do que sua versão resumida, cujo escore total atinge 15 pontos. Outros estudos mostraram, ainda, que ela oferece medidas válidas e confiáveis para idosos com DP21-24. Descrita em língua inglesa por Yesavage et al. ${ }^{19}$, a escala foi desenvolvida especialmente para o rastreamento dos transtornos de humor, com perguntas que não se restringem à esfera das queixas somáticas. Dentre as suas vantagens, destacam-se o fato de poder ser autoaplicada pelo próprio participante ou aplicada por um entrevistador treinado, sendo composta por perguntas fáceis de serem compreendidas, com pouca variação nas possibilidades de respostas.

Todas as entrevistas e coletas de dados foram realizadas no Ambulatório de Fisioterapia Neurológica da Universidade Estadual de Goiás. Dois avaliadores foram treinados para aplicação dos testes e questionários utilizados, mantendo-se "cegos" quanto aos grupos em que os participantes se encontravam.

No que se refere à análise dos dados, a estatística descritiva (média e erro-padrão) foi utilizada para caracterização dos resultados. Pelos testes de normalidade de Shapiro-Wilk e homogeneidade de Levene, foi indicada a utilização da estatística paramétrica. Diante disso, foram aplicados os testes de análise de variância para medidas repetidas (ANOVA de duas vias), tendo como fatores "grupo" e "momento" (antes e depois do tratamento), e o teste $t$ de Student para amostras independentes, para verificar as características iniciais dos grupos. Em todas as análises foi considerado um nível de significância de 5\% $(P<0,05)$.

Esta pesquisa recebeu aprovação do Comitê de Ética do Hospital de Goiânia. No referido processo, consta uma descrição dos propósitos e das hipóteses a serem testadas, a análise crítica dos riscos e benefícios envolvendo os sujeitos da pesquisa, a explicação das responsabilidades do pesquisador, da instituição e do promotor e uma declaração de que os resultados serão tornados públicos (sejam eles favoráveis ou não). Os participantes assinaram um Termo de Consentimento Livre e Esclarecido baseado nas normas estabelecidas pela Resolução no 196/96, do Conselho Nacional de Saúde, para as pesquisas envolvendo seres humanos.

\section{RESULTADOS}

Este estudo foi formado por 26 idosos diagnosticados com DP idiopática, divididos entre os grupos GE e GC. Na Tabela 1 
encontram-se as características de ambos os grupos em relação a idade, gênero, nível de escolaridade, tempo de diagnóstico e grau de acometimento da doença. O teste $t$ de Student para amostras independentes apontou que os grupos são similares no que se refere aos escores de tais variáveis dependentes $(p>0,05)$.

Tabela 1. Análise descritiva da amostra

\begin{tabular}{lccc}
\hline Caracteristica & $\begin{array}{c}\text { Grupo } \\
\text { Experimental }\end{array}$ & Grupo Controle & Significância \\
\hline Gênero (Masc.: Fem.) & $8: 5$ & $6: 7$ & 0,45 \\
Idade (anos) & $67,1 \pm 1,2$ & $67,7 \pm 1,5$ & 0,66 \\
Escolaridade (anos) & $5,7 \pm 2,4$ & $4,8 \pm 1,1$ & 0,19 \\
Tempo do diagnóstico (anos) & $3,8 \pm 0,9$ & $4,2 \pm 0,7$ & 0,11 \\
Escala de Hoehn-Yarh & $2,58 \pm 0,14$ & $2,66 \pm 0,15$ & 0,47 \\
\hline
\end{tabular}

Idade, escolaridade, tempo de diagnóstico e gravidade da doença foram expressos em média e erro-padrão.

Em relação aos valores obtidos pelos grupos GE e GC na EDG, observamos uma diminuição dos sintomas depressivos nos sujeitos participantes do tratamento fisioterápico em relação àqueles que compuseram o GC (Figura 1). No entanto, a análise obtida pela ANOVA de duas vias para medidas repetidas não foi significativa para a interação "grupo" X "momento", apontando uma tendência de significância sob um erro alfa de $6 \%$ em um contraste linear de dados ( $F=3,923$; $p=0,060)$. A análise apontou, ainda, semelhança estatística em relação aos fatores "grupo" ( $F=0,240 ; p=0,879)$ e "momento" ( $F=2,207 ; p=0,152)$.

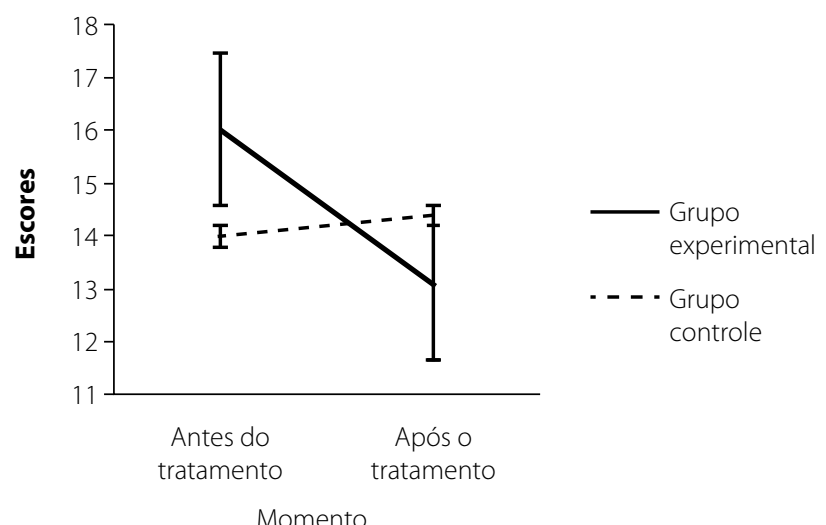

Figura 1. Escore dos pacientes dos grupos experimental e controle na Escala de Depressão Geriátrica. Os dados foram expressos em média e erro-padrão.

Sobre a medicação, todos os sujeitos (100,00\%) faziam uso de medicação precursora de dopamina (L-Dopa). Segundo informações coletadas, tais medicações haviam sido inseridas no mínimo seis meses antes do início do tratamento cognitivo-motor. Nenhum participante fazia uso de antidepressivos no início da abordagem cognitivo-motora.
Durante o período de tratamento, dois sujeitos $(7,69 \%$ da amostra) foram excluídos, por ter havido a inclusão de medicação inibidora seletiva de recaptação de serotonina. Tal exclusão se deu diante da uma possível interferência do fármaco no resultado da abordagem cognitivo-motora proposta. Nenhum participante relatou desconforto (físico ou mental) durante a terapia realizada.

\section{DISCUSSÃO}

O presente estudo objetivou analisar a eficácia de uma intervenção fisioterápica com estimulação cognitivo-motora em idosos diagnosticados com DP idiopática. Após seis meses de tratamento, observamos uma diminuição dos sintomas depressivos no GE, mas sem diferença significativa quando comparado aos resultados do GC.

É fato que toda comprovação científica almejada requer um rigor metodológico exemplar. Ao se realizar qualquer pesquisa, é desejo comum dos pesquisadores que a hipótese nula - aquela relacionada à igualdade de resultados não seja encontrada. Nesse raciocínio, a comunidade científica biomédica admite, como significância estatística, todo e qualquer resultado cujo erro alfa encontrado seja menor que $5 \%$ ( $p<0,050)$, ou, em casos mais peculiares, menor que $1 \%(p<0,001)$. Pela análise fria dos resultados, poderíamos afirmar que, em relação à terapia proposta, não há diferença ao paciente, caso este venha ou não a realizar a referida assistência.

No entanto, é importante dizer que encontramos uma diminuição dos sintomas depressivos no GE, sob um nível de significância de $6 \%$, em um contraste linear dos dados ( $p$ $=0,060$ ). Como o valor encontrado no erro alfa - envolvendo a possível admissão de casos falso-positivos - foi muito próximo à exigência científica supracitada, alguns estatísticos consideram tal resultado importante, classificando-o como "tendência significativa" (situação em que os valores constatados encontram-se entre $0,050<p<0,070$ e, muitas vezes, estão relacionados a pequenos fatores que impossibilitaram alcançar um intervalo de confiança maior que 95\%). Hackshaw e Kirkwood ${ }^{25}$ apoiam tal discussão alegando que o valor de 5\% como índice de confiabilidade foi proposto por Ronald Aylmer Fisher em 1925, não devendo ser analisado como um ponto fixo e imutável de delimitação das hipóteses nula e alternativa.

Nessa perspectiva, cabe refletirmos sobre alguns fatores que tentamos controlar neste estudo e que talvez possam ter influenciado no nível de significância encontrado. Primeiramente, há uma relação inversamente proporcional existente entre o tamanho amostral e o erro alfa. Em nosso estudo conseguimos analisar apenas 13 idosos do GE e 13 do GC. Caso tivéssemos uma amostra maior, é possível que o resultado apresentasse melhor significância. Entre- 
tanto, a exigência de comparecimento de três vezes na semana impediu que aumentássemos o número de sujeitos da amostra. Em segundo lugar, é possível que, caso o tempo de tratamento tivesse se estendido a um ano, teríamos obtido resultados significativamente melhores do que os encontrados. Entretanto, ainda que consideremos que os resultados de seis meses de terapia não tenham sidos positivos, como conseguiríamos discutir o baixo índice de desistência ocorrido nesse período (este propiciado ainda por uma alteração na medicação)? Tal dado é um indicativo de que a terapia proposta estava sendo importante e reconhecida pelos pacientes. Em terceiro lugar, outro fator a ser analisado é a aplicação de questionários, utilizados para quantificar as variáveis dependentes. É fato que questionários apresentam desvantagens, pois muitos restringem as análises dos dados, pelas características discretas que possuem. No entanto, como dito anteriormente, a EDG é altamente reconhecida na literatura científica, com comprovada aplicação na DP21-24. Além do mais, por meio dos testes de normalidade, homogeneidade e esfericidade, foi-nos habilitado o uso da estatística paramétrica para os dados em questão.

Estudos comprovam que o exercício físico exerce importante fator neuroprotetor celular, agindo tanto nos sintomas motores quanto nos sintomas psiquiátricos de idosos 26,27 . Mecanismos neurobiológicos associados a uma prática constante de abordagem motora podem promover a ativação de diversos neurotransmissores (como acetilcolina, norepinefrina, serotonona e GABA), além de estimular a neurogênese e a proliferação do fator neurotrófico derivado do cérebro ${ }^{28}$. Assim, ao realizar o tratamento fisioterápico com estimulação cognitivo-motora, desejávamos observar indiretamente tais efeitos, por meio dos valores encontrados na EDG.

O diferencial de nosso estudo está na abordagem aplicada aos pacientes por meio de tarefas duplas (cognitivo + motora) em grupo. Diante disso, os resultados encontrados podem ser arguidos do ponto de vista físico ou psicológico. Se de um lado os exercícios aplicados poderiam propiciar uma melhora na qualidade de vida dos sujeitos, por outro a atividade lúdica realizada em grupo, sobretudo com a presença de cuidadores e familiares, fortalece a melhora psicológica de bem-estar e humor do paciente, promovendo resultados significativos nos sintomas depressivos.

Ainda no que se refere ao tratamento aplicado, a padronização de três sessões semanais com objetivos diferentes visou não tornar a terapia uma rotina cansativa ao paciente. A utilização de atividades cognitivo-motoras se deu pelo fato de, apesar de a DP ser uma doença eminentemente motora, haver uma grande prevalência de distúrbios psíquico-comportamentais nessa população ${ }^{13}$. Diante disso, por meio de atividades realizadas com materiais e objetos de texturas e pesos específicos, pretendemos estimular algumas funções corticais superioras como a praxia, a gnosia, a atenção con- centrada, a memória, o raciocínio, as funções visuoperceptivas, visuoconstrutivas e executivas, entre outras.

Christofoletti et al. ${ }^{29}$ observaram uma importante alteração na qualidade de vida de sujeitos com DP, muitas vezes potencializada pelo quadro de exclusão social ao qual o paciente é submetido. Margis et al..$^{30}$ corroboram o estudo anterior alegando que a alteração na qualidade de vida de pacientes com DP usualmente está associada à alta prevalência de sintomas depressivos na população em questão e ao estigma negativo propiciado pela doença. Há evidência de que a abordagem em grupo traga benefícios além do propiciado pela terapia, podendo ser ocasionado pela ampliação das redes sociais dos pacientes ${ }^{31}$. Nessa realidade, por meio da realização de atividades em grupo, forçamos a interação social de pacientes com DP, promovendo a diminuição dos sintomas depressivos pela ampliação do convívio social.

Optamos por restringir o foco deste estudo aos sintomas depressivos em vez de casos com depressão maior, em decorrência da alta prevalência de tais sintomas em pacientes com DP32. Além disso, os pacientes diagnosticados com depressão maior comumente fazem uso de medicação antidepressiva, em que muitas vezes há incertezas a respeito do tempo que é necessário para que o fármaco comece a agir beneficamente no organismo humano ${ }^{33}$. Assim sendo, teríamos um grave problema metodológico, causado pela seleção de pacientes que fazem uso de dosagens díspares de medicação, cuja ingestão teria se iniciado em diferentes momentos, impedindo a tentativa de comprovação de qualquer benefício causado pela terapia cognitivo-motora em si.

Sobre a discussão anterior, futuros estudos podem se concentrar na análise de idosos com DP diagnosticados com depressão maior, desde que se consiga controlar todos os vieses metodológicos (como fármaco utilizado, princípio ativo, dosagem, início da administração medicamentosa e outros). Apesar de concordamos com tal argumento, não realizamos esse feito, pois acreditamos que restringir por demasia a amostra trará desvantagens, por não representar os casos heterogêneos genuínos na comunidade, impedindo, por conseguinte, a generalização dos resultados a pacientes com DP.

A principal limitação de nosso artigo está relacionada ao número de sujeitos incluídos em nosso trabalho. Conquanto possa ser arguido sobre o pequeno tamanho amostral, há que se considerar a dificuldade de recrutamento dos sujeitos, bem como o fato de a DP apresentar um diagnóstico complexo, exigindo a integração da avaliação clínica com exames laboratoriais e de neuroimagem, nem sempre disponíveis. Na DP, a grande dificuldade de diagnóstico está em se diferenciar a DP idiopática das síndromes parkinsonianas secundárias ao comprometimento cerebral.

Por fim, é importante dizer que protocolo proposto apresentou boa aceitação pelos pacientes com DP, conforme evidenciado pelo baixo índice de perdas amostrais. Em estudo 
envolvendo sequência semelhante de exercícios, obteve-se uma melhora significativa dos pacientes com DP em relação ao equilíbrio humano ${ }^{34}$.

Em conclusão, os resultados de nosso estudo refletem um potencial do referido protocolo, tendo obtido uma diminuição quase significativa dos sintomas depressivos de pacientes diagnosticados com DP. Apesar de haver a necessidade de elaboração de mais estudos envolvendo a temática da ação fisioterápica nos sintomas depressivos de pacientes com DP, este artigo conseguiu alcançar o objetivo de promover tal discussão, muitas vezes negligenciada por profissionais da saúde que assistem os pacientes.

\section{REFERÊNCIAS}

1. Lau LM, Breteler MM. Epidemiology of Parkinson's disease. Lancet. 2006;5:525-35.

2. Calne D. A definition of Parkinson's disease. Parkinsonism Relat Disord. 2005;11:S39-40.

3. Calabresi P, Picconi B, Parnetti L, Di Filippo M. A convergent model for cognitive dysfunction in Parkinson's disease: the critical dopamine-acetylcholine synaptic balance. Lancet. 2006:5:974-83.

4. Obeso JA, Rodríguez-Oroz MC, Benitez-Temino B, Blesa FJ, Guridi J, Marin C, et al. Functional organization of the basal ganglia: therapeutic implications for Parkinson's disease. Mov Disord. 2008;23:S548-59.

5. Zimprich A, Biskup S, Leitner $P$, Lichtner $P$, Farrer $M$, Lincoln $S$, et al. Mutations in LRRK2 cause autosomal-dominant parkinsonism with pleomorphic pathology. Neuron. 2004:44:601-7.

6. McKinlay A, Grace RC, Dalrymple-Alford JC, Anderson T, Fink J, Roger D. A profile of neuropsychiatric problems and their relationship to quality of life for Parkinson's disease patients without dementia. Parkinsonism Relat Disord. 2008;14(1):37-42.

7. Reijnders JS, Ehrt U, Weber WE, Aarsland D, Leentjens AF. A systematic review of prevalence studies of depression in Parkinson's disease. Mov Disord. 2008;23:183-9.

8. Stella F, Banzato CE, Quagliato EM, Viana MA, Christofoletti G. Psychopatological features in patients with Parkinson's disease and caregiver's burden. Int J Geriatr Psychiatry. 2009;24(10):1158-65.

9. Cimino CR, Sifers CA, Zesiewicz TA. Depressive symptoms in Parkinson disease: degree of association and rate of agreement of clinician-based and self-report measures. J Geriatr Psychiatry Neurol. 2011;24(4):199-205.

10. Dickson DW, Fujishiro H, Orr C, DelleDonne A, Josephs KA, Frigerio R, et al. Neuropathology of non-motor features of Parkinson disease. Parkinsonism Relat Disord. 2009;15(Suppl 3):S1-5.

11. Deslandes A, Hernandez SSS, Gobbi S, Costa JLR, Stella F. Exercise and mental health: many reasons to move. Neuropsychobiology. 2009;59(4):191-8.

12. Hughes AJ, Daniel SE, Kilford L, Lees AJ. Accuracy of clinical diagnosis of idiopathic Parkinson's disease: a clinico-pathological study of 100 cases. J Neurol Neurosurg Psychiatry. 1992;55:181-4.

13. Hoehn MM, Yahr MD. Parkinsonism: onset, progression and mortality. Neurology. 1967;17(5):427-42

14. Martinez-Martín P, Gil-Nagel A, Gracia LM, Gómez JB, Martínez-Sarriés J, Bermejo F. Unified Parkinson's disease rating scale: characteristics and structure. Mov Disord. 1994;9(1):76-83.
15. Folstein MF, Folstein SE, Mchugh PR. Mini-Mental State: a practical method for grading the cognitive state of patients for the clinician. J Psychiatr Res. 1975;12:189-98.

16. Nitrini R, Lefèvre BH, Mathias SC, Caramelli P, Carrilho PE, Sauaia N, et al. Neuropsychological tests of simple application for dementia diagnosis. Arq Neuropsiquiatr. 1994;52:634-8.

17. Sunderland T, Hill JL, Melow AM, Lawlor BA, Gundersheimer J, Grafman JH. Clock drawing in Alzheimer's disease: a novel measure of dementia severity. J Am Geriatr Soc. 1989;37:725-9.

18. DSM-IV-TR - Manual Diagnóstico e Estatístico de Transtornos Mentais. 4. ed. Porto Alegre: Artmed; 2002

19. Yesavage JA, Brink TL, Rose TL, Lum 0, Huang V, Adey M, et al. Development and validation of a geriatric depression screening scale: a preliminary report. J Psychiat Res. 1983;17(1):37-49.

20. Souza RL, Medeiros JGM, Moura ACL, Souza CLM, Moreira IF. Validade e fidedignidade da Escala de Depressão Geriátrica na identificação de idosos deprimidos em um hospital geral. J Bras Psiquiatr. 2007;56(2):102-7.

21. Tumas V, Rodrigues GGR, Farias TLA, Crippa JAS. The accuracy of diagnosis of major depression in patients with Parkinson's disease: a comparative study among the UPDRS, the geriatric depression scale and the Beck Depression Inventory. Arq Neuropsiquiatr. 2008;66(2):152-6.

22. Montorio IIM. The geriatric depression scale: a review of its development and utility. Int Psychogeriatr. 1996:8(1):103-12

23. Paradela EMP, Lourenço RA, Veras RP. Validation of Geriatric Depression Scale in general outpatient clinic. Rev Saude Publica. 2005;39(6):918-23.

24. Thompson AW, Liu H, Hays RD, Katon WJ, Rausch R, Diaz N, et al. Diagnostic accuracy and agreement across three depression assessment measures for Parkinson's disease. Parkinsonism Relat Dis. 2011;17(1):40-5.

25. Hackshaw A, Kirkwood A. Interpreting and reporting clinical trials with results of borderline significance. BMJ. 2011;343. In press. D0I: 10.1136/bmj.d3340.

26. Peluso MA, Guerra de Andrade LH. Physical activity and mental health: the association between exercise and mood. Clinics. 2005;60(1):61-70.

27. Speelman AD, Van de Warrenburg BP, Van Nimwegen M, Petzinger GM, Munneke M, Bloem BR. How might physical activity benefit patients with Parkinson disease? Nat Rev Neurol. 2011;7(9):524-34

28. Ma Q. Beneficial effects of moderate voluntary physical exercise and its biological mechanisms on brain health. Neurosci Bull. 2008;24(4):265-70.

29. Christofoletti G, Formiga CKMR, Borges G, Stella F, Damasceno BP. Aspectos físicos e mentais na qualidade de vida de pacientes com doença de Parkinson idiopática. Fisioter Pesq. 2009;16(1):65-9.

30. Margis R, Donis KC, Schonwald SV, Rieder CRM. WHOQOL-OLD assessment of quality of life with Parkinson' disease: influence of sleep and depressive symptoms. Rev Bras Psiquiatr. 2010;32(2):125-31

31. States RA, Spierer DK, Salem Y. Long-term group exercise for people with Parkinson's disease: a feasibility study. J Neurol Phys Ther. 2011;35(3):122-8.

32. Riedel O, Klotsche J, Spottke A, Deuschl G, Förstl H, Henn F, et al. Frequency of dementia, depression, and other neuropsychiatric symptoms in 1,449 outpatients with Parkinson's disease. J Neurol. 2010;257(7):1073-82.

33. Barone B. Treatment of depressive symptoms in Parkinson's disease. Eur J Neurol. 2011:18(1):11-5.

34. Christofoletti G, Freitas RT, Cândido ER, Cardoso CS. Eficácia de tratamento fisioterapêutico no equilíbrio estático e dinâmico de pacientes com doença de Parkinson. Fisioter Pesq. 2010;17(3):259-63. 